\title{
Conversations and Reactions Around Severe Hypoglycemia (CRASH): Japan Results From a Global Survey of People with T1DM or Insulin-Treated T2DM and Caregivers
}

Toshiyuki Iwahori · Frank Snoek · Yukiko Nagai · Erik Spaepen •

Beth D. Mitchell · Mark Peyrot

Received: September 3, 2021 / Accepted: January 24, 2022 / Published online: February 24, 2022

(c) The Author(s) 2022

\section{ABSTRACT}

Aims: The CRASH study examined severe hypoglycemia $(\mathrm{SH})$ experiences among people with diabetes (PWD) and caregivers across eight countries. Here we report findings from the Japan cohort, with references to data from the United Kingdom (UK) cohort.

Materials and Methods: Adults with type 1 (T1DM) or insulin-treated type 2 diabetes mellitus (T2DM) and caregivers (not necessarily related) were recruited from online patient panels. Participants who had experienced at least one SH event in the past 3 years were eligible for study inclusion. Participants

T. Iwahori · Y. Nagai

Eli Lilly Japan, K.K., Kobe, Hyogo, Japan

F. Snoek

Department of Medical Psychology, Amsterdam University Medical Centers, Vrije Universiteit

Amsterdam, Amsterdam, The Netherlands

E. Spaepen

HaaPACS GmbH, Schriesheim, Germany

B. D. Mitchell

Eli Lilly \& Company, Indianapolis, IN, USA

M. Peyrot

Department of Sociology, Loyola University

Maryland, Baltimore, MD, USA

Y. Nagai $(\varangle)$

5-1-28 Isogamidori, Chuo-Ku, Kobe 651-0086, Japan

e-mail: nagai_yukiko@lilly.com completed an online survey regarding their experience with $\mathrm{SH}$, its treatment, and actions during and after an event.

Results: Of the 9367 PWD and caregivers from the online patient panels, 8475 participants were ineligible and a total of 53 Japanese participants (35 T1DM, 9 T2DM, 9 caregivers) completed the survey. Most SH incidents occurred at home and were unattended by a healthcare provider. For T1DM, 29\% of Japan PWD and $13 \%$ of the UK PWD called an ambulance during an SH event; of these, 90\% (Japan) and 50\% (UK) were transported to hospital. Glucagon use was low (3\% Japan and 10\% UK for T1DM). Japanese respondents reported emotional impacts of $\mathrm{SH}$, including feeling scared (86\% T1DM, 56\% T2DM), unprepared (63\% T1DM, 78\% T2DM), and helpless (60\% T1DM, 33\% T2DM). Despite the emotional burden, most PWD did not immediately discuss their $\mathrm{SH}$ event with a healthcare provider, with the majority (75\% T1DM, 71\% T2DM) waiting until their next doctor's appointment.

Conclusion: Conversations around $\mathrm{SH}$ between healthcare providers and PWD appear to be insufficient in Japan. An emotional burden of SH was reported by PWD and caregivers. Education regarding the prevention of $\mathrm{SH}$ and available treatment options may reduce $\mathrm{SH}$ events and improve treatment preparation, while alleviating PWD concerns. 
Keywords: Caregivers; Diabetes; Emotional burden; Glucagon; Japan; Severe hypoglycemia

\section{Key Summary Points}

Why carry out this study?

Severe hypoglycemia (SH) is an acute event requiring assistance from another person to take corrective actions, and places considerable burden on people with diabetes (PWD) both physically and in terms of psychological well-being, diabetes-related distress, and fear of further hypoglycemia events

The Conversations and Reactions Around Severe Hypoglycemia (CRASH) study examined severe hypoglycemia $(\mathrm{SH})$ experiences among people with T1DM or insulin-treated T2DM and caregivers (CGs) across eight countries

We report the experiences and treatments of SH events among Japanese PWD and CGs from the CRASH study, the first study to directly recruit patients who experienced hypoglycemia in any Asian population

\section{What was learned from the study?}

In Japanese PWD and CGs, our findings suggest that many $\mathrm{SH}$ events occur at home, many PWD and CGs are highly affected emotionally by SH events, effective communication between PWD and healthcare providers appears to be lacking, and ambulance service is commonly used in an SH event but few PWD use glucagon as rescue therapy

Education regarding $\mathrm{SH}$ treatment options may improve treatment preparation for $\mathrm{SH}$ events and may help alleviate patient concerns

\section{INTRODUCTION}

For people with diabetes (PWD), hypoglycemia is a common side effect of glucose-lowering treatments such as insulin and sulfonylureas, presenting a major barrier to achieving satisfactory long-term glycemic control. It is well established that glucose control and variability, and the risk of hypoglycemia, are closely related [1].

Hypoglycemia causes significant morbidity, characterized by acute symptoms including palpitations, perspiration, confusion, dizziness, headache, and blurred vision [2]. Severe hypoglycemia $(\mathrm{SH})$ requires assistance from another person to actively administer carbohydrates, glucagon, or take other corrective actions $[3,4]$. Beyond the immediate symptoms, $\mathrm{SH}$ increases the risk of cardiovascular events [5-7], dementia [8], and overall mortality [7, 9]. SH affects both people with type 1 diabetes mellitus (T1DM) and those with type 2 diabetes mellitus (T2DM) being treated with insulin and other antihyperglycemic medications. SH also contributes to fear of glucose-lowering treatment, which is a barrier to achieving optimal glucose control [10]. It is therefore imperative that healthcare providers (HCPs) and PWD are mindful of the risks and impact of hypoglycemia.

A report by the Japanese Diabetes Society between 2014 and 2015 revealed that 20,000 PWD in Japan are estimated to be transported annually for emergency treatment of SH [3]. Whilst SH was observed in all age brackets for Japanese patients with T1DM, the majority of $\mathrm{SH}$ cases for Japanese patients with T2DM occurred in those aged 65 years or older [3]. In addition, the increasing number of people diagnosed with T2DM in Japan over the past few decades is regarded as a public health issue, with prevalence continuing to increase mostly due to an aging population [11]. In turn, this is likely to increase the number of people taking insulin or other medications to lower blood glucose, potentially increasing the incidence of hypoglycemia events. Thus, raising awareness of the importance of early intervention and strategies to prevent and treat $\mathrm{SH}$ is warranted for patient safety, and to reduce the economic 
burden of emergency transportation and hospitalization [12].

Aside from the physical effects of $\mathrm{SH}$, these events place considerable burden on PWD in terms of psychological well-being, diabetes-related distress, and fear of further hypoglycemia events [13]. Minimization of SH risk is likely to contribute to improvements in quality of life for PWD as well as their caregivers (CGs). However, reports among East Asian populations concerning $\mathrm{SH}$ remain scarce, with real-world data surrounding patient circumstances largely unknown.

The Conversations and Reactions Around Severe Hypoglycemia (CRASH) study was designed to increase understanding of the experiences, behaviors, and impacts of $\mathrm{SH}$ on persons with T1DM and insulin-treated T2DM, as well as CGs of PWD. We report the experiences and treatments of SH events among Japanese PWD with T1DM or insulin-treated T2DM, and CGs from the CRASH study which was the first study to directly recruit patients who experienced hypoglycemia in any Asian population.

\section{METHODS}

\section{Study Design and Participant Enrollment}

The study design has been previously described [14]. Briefly, CRASH was a cross-sectional, online survey conducted in eight countries (Canada, China, France, Germany, Japan, Spain, USA, UK).

PWD were recruited from online patient panels and were eligible for study inclusion if the following self-reported criteria were met: aged at least 18 years at the time of screening, diagnosed with T1DM or T2DM and treated with insulin via injection or pump at the time of study commencement, experienced one or more SH events in the prior 3 years while treated with insulin, and treated with insulin in combination with oral or injectable diabetes treatments except sulfonylureas. CGs were laypersons who provided care for PWD aged at least 4 years who met the above criteria for hypoglycemia and treatment and were relied upon in the event of an episode of SH. CGs and PWD were recruited separately and were not analyzed as dyads. Persons were excluded from the study if they had schizophrenia or bipolar disorder, or were professionally trained HCPs, employees of pharmaceutical companies, or had completed any other type of online survey in the 2 months prior.

We report the findings from the Japan cohort, with references to data from the CRASH UK cohort. While citizens in Japan and the UK pay tax for health insurance which includes ambulance transportation, ambulance services are also provided free of charge as part of the national healthcare in both countries. This contrasts with many other nations where there are additional fees for ambulance use.

Ethical approval was received from Clinical Research Promotion Network Japan IRB (Osaka, Japan) and all participants provided informed consent. This study was performed in accordance with the Helsinki Declaration of 1964 and its later amendments.

\section{Survey Content}

Participants were given the following definition of severe hypoglycemia: "Severe hypoglycemia is a low blood sugar event that [you/the person with diabetes] cannot treat by [yourself/themselves]. This means a low blood sugar emergency during which [you/the person with diabetes] might have nearly lost consciousness, experienced seizures, or had a coma. During such an event, [you/the person with diabetes] will need help from another person to recover."

Each participant completed questions regarding demographics; current diabetes management; recent history and awareness of hypoglycemia; the setting, symptoms, actions taken; and emotional impact of the most recent SH episode. Participants were also asked questions relating to conversations with HCPs prior and subsequent to the most recent $\mathrm{SH}$ event.

\section{Statistical Analyses}

Descriptive data were analyzed using SAS statistical software version 9.4 (The SAS Institute, 
Cary NC). Categorical data are presented as counts and percentages. Continuous data are presented as mean \pm standard deviation.

\section{RESULTS}

\section{Baseline Demographics and Clinical Characteristics}

Of 9367 people screened from patient panels, 8475 were determined to be ineligible (did not meet eligibility criteria) to complete the survey and 839 did not fully complete the survey (Fig. 1). There were 5173 respondents with T1DM or T2DM who proceeded to screening, and 4844 reported no SH events. Study ineligibility was mostly due to participants not experiencing an $\mathrm{SH}$ event (56.8\%), not having a diabetes diagnosis $(26.0 \%)$, or having diabetes other than T1DM or T2DM (8.3\%). Overall, 53 Japanese participants completed the survey. We report data collected from 53 Japanese participants, including 44 PWD (35 T1DM, 9 T2DM) and 9 CGs ( 3 provided care for patients with T1DM and 6 for patients with T2DM). Data from 380 UK participants were used as a contrasting cohort for clinical characteristics (PWD and CGs), most recent SH event, glucagon use, and the effects of SH on multiple life domains (PWD and CGs). The characteristics of the participants are reported in Tables 1 and 2.

Mean age for Japanese PWD surveyed was $49.9 \pm 9.2$ years $(\mathrm{T} 1 \mathrm{DM})$ and $50.9 \pm 11.2$ years (T2DM). For T1DM, there was a similar proportion of men and women, but there were fewer female participants with T2DM $(48.6 \%$ and $22.2 \%$, respectively). The mean time since diagnosis was $25.0 \pm 11.8$ years for participants with T1DM, and $12.8 \pm 8.0$ for those with T2DM. In terms of diabetes management, most Japanese PWD reported their most recent HbA1c level was $\leq 7 \%(57.1 \%$ T1DM, $22.2 \%$ $\mathrm{T} 2 \mathrm{DM})$ or between $7.1 \%$ and $9 \%$ (34.3\% T1DM, $66.6 \%$ T2DM) (Table 1).

Age (T1DM) and sex (T1DM and T2DM) were comparable between the Japan and UK cohorts. However, most recent laboratory HbA1c, body mass index, and number of SH events (T2DM) were numerically lower for the Japan PWD in comparison to the UK PWD. The Japan PWD frequently reported impaired hypoglycemia

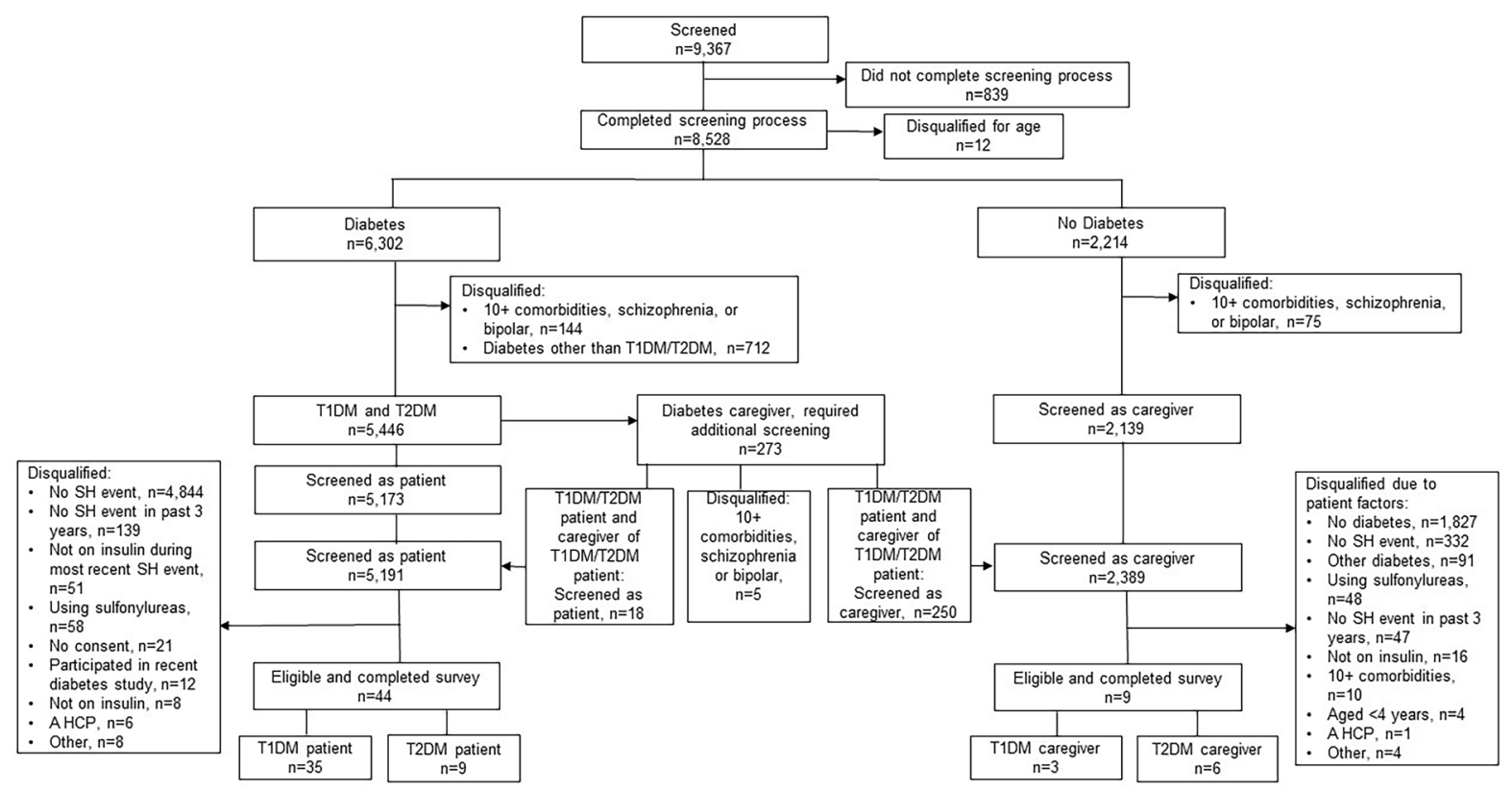

Fig. 1 Patient disposition flowchart. HCP healthcare provider, $n$ number of participants, $S H$ severe hypoglycemia, TIDM type 1 diabetes mellitus, T2DM type 2 diabetes mellitus 
Table 1 Baseline demographic and clinical characteristics of PWD in Japan and the UK

\begin{tabular}{|c|c|c|c|c|}
\hline & \multicolumn{2}{|c|}{ Japanese PWD $(n=44)$} & \multicolumn{2}{|c|}{ UK PWD $(n=208)$} \\
\hline & $\begin{array}{l}\text { T1DM } \\
(n=35)\end{array}$ & $\begin{array}{l}\text { T2DM } \\
(n=9)\end{array}$ & $\begin{array}{l}\text { T1DM } \\
(n=110)\end{array}$ & $\begin{array}{l}\text { T2DM } \\
(n=98)\end{array}$ \\
\hline Age, mean years $(\mathrm{SD})$ & $49.9(9.2)$ & $50.9(11.2)$ & $53.4(12.5)$ & $58.3(11.4)$ \\
\hline Female, $n(\%)$ & $17(48.6)$ & $2(22.2)$ & $50(45.5)$ & $24(24.5)$ \\
\hline Body mass index, $\mathrm{kg} / \mathrm{m}^{2}$, mean $(\mathrm{SD})$ & $21.3(3.9)$ & $24.4(4.8)$ & $28.3(10.2)$ & $34.1(10.6)$ \\
\hline Time since diagnosis, mean years $(\mathrm{SD})$ & $25.0(11.8)$ & $12.8(8.0)$ & $28.6(15.4)$ & $14.2(7.8)$ \\
\hline Used insulin for $>5$ years, $n(\%)$ & - & $5(55.6)$ & - & $49(50.0)$ \\
\hline Ever prescribed a glucagon kit, $n(\%)$ & $6(17.1)$ & $0(0.0)$ & $52(47.3)$ & $8(8.2)$ \\
\hline $\begin{array}{l}\text { Impaired hypoglycemia awareness (Gold score } \geq 4 \text { ) } \\
n(\%)\end{array}$ & $12(34.3)$ & $5(55.6)$ & $33(30.0)$ & $15(15.3)$ \\
\hline \multicolumn{5}{|l|}{ Most recent laboratory $\mathrm{HbAlc}, n(\%)$} \\
\hline$<6.5 \%(48 \mathrm{mmol} / \mathrm{mol})$ & $13(37.1)$ & $0(0.0)$ & $16(14.5)$ & $15(15.3)$ \\
\hline $6.5-7.0 \%(48-53 \mathrm{mmol} / \mathrm{mol})$ & $7(20.0)$ & $2(22.2)$ & $26(23.6)$ & $17(17.3)$ \\
\hline $7.1-8 \%(54-64 \mathrm{mmol} / \mathrm{mol})$ & $8(22.9)$ & $3(33.3)$ & $33(30.0)$ & $25(25.5)$ \\
\hline $8.1-9 \%(65-75 \mathrm{mmol} / \mathrm{mol})$ & $4(11.4)$ & $3(33.3)$ & $12(10.9)$ & $12(12.2)$ \\
\hline$>9.1 \%(>75 \mathrm{mmol} / \mathrm{mol})$ & $2(5.7)$ & $0(0.0)$ & $15(13.7)$ & $10(10.2)$ \\
\hline Unknown & $1(2.9)$ & $1(11.1)$ & $8(7.3)$ & $19(19.4)$ \\
\hline \multicolumn{5}{|l|}{ Hypoglycemia events } \\
\hline In the past 12 months, mean (SD) & $1.5(2.3)$ & $0.8(1.1)$ & $3.3(10.0)$ & $2.2(3.6)$ \\
\hline In the past 12 months, median (range) & $1(0,10)$ & $0(0,3)$ & $1(0,100)$ & $1(0,24)$ \\
\hline In the past 3 years, mean $(\mathrm{SD})$ & $4.3(6.9)$ & $2.0(1.3)$ & $7.0(13.1)$ & $5.1(9.9)$ \\
\hline In the past 3 years, median (range) & $2(1,30)$ & $2(1,5)$ & $2(1,100)$ & $2(1,80)$ \\
\hline \multicolumn{5}{|c|}{ Number of severe hypoglycemia events in the past 12 months, $n$ (\%) } \\
\hline 0 & $10(28.6)$ & $5(55.6)$ & $16(14.5)$ & $15(15.3)$ \\
\hline 1 & $17(48.6)$ & $2(22.2)$ & $52(47.3)$ & $47(48.0)$ \\
\hline 2 & $4(11.4)$ & $1(11.1)$ & $19(17.3)$ & $18(18.4)$ \\
\hline$\geq 3$ & $4(11.4)$ & $1(11.1)$ & $23(20.9)$ & $18(18.4)$ \\
\hline \multicolumn{5}{|l|}{ Comorbid conditions, $n$ (\%) } \\
\hline Allergies & $4(11.4)$ & $0(0.0)$ & $13(11.8)$ & $5(5.1)$ \\
\hline Anxiety & $1(2.9)$ & $0(0.0)$ & $25(22.7)$ & $23(23.5)$ \\
\hline Arthritis & $2(5.7)$ & $0(0.0)$ & $12(10.9)$ & $21(21.4)$ \\
\hline Cancer & $1(2.9)$ & $1(11.1)$ & $5(4.5)$ & $1(1.0)$ \\
\hline Chest pain & $1(2.9)$ & $0(0.0)$ & $5(4.5)$ & $4(4.1)$ \\
\hline
\end{tabular}


Table 1 continued

\begin{tabular}{|c|c|c|c|c|}
\hline & \multicolumn{2}{|c|}{ Japanese PWD $(n=44)$} & \multicolumn{2}{|c|}{ UK PWD $(n=208)$} \\
\hline & $\begin{array}{l}\text { T1DM } \\
(n=35)\end{array}$ & $\begin{array}{l}\text { T2DM } \\
(n=9)\end{array}$ & $\begin{array}{l}\text { T1DM } \\
(n=110)\end{array}$ & $\begin{array}{l}\text { T2DM } \\
(n=98)\end{array}$ \\
\hline Chronic kidney disease & $3(8.6)$ & $0(0.0)$ & $6(5.5)$ & $8(8.2)$ \\
\hline Depression & $3(8.6)$ & $0(0.0)$ & $22(20.0)$ & $29(29.6)$ \\
\hline Gastroesophageal reflux disease & $1(2.9)$ & $0(0.0)$ & $5(4.5)$ & $8(8.2)$ \\
\hline Heart arrhythmias & $2(5.7)$ & $0(0.0)$ & $4(3.6)$ & $6(6.1)$ \\
\hline High cholesterol & $4(11.4)$ & $1(11.1)$ & $30(27.3)$ & $40(40.8)$ \\
\hline Hypertension & $10(28.6)$ & $3(33.3)$ & $20(18.2)$ & $30(30.6)$ \\
\hline Migraine headaches & $3(8.6)$ & $0(0.0)$ & $6(5.5)$ & $6(6.1)$ \\
\hline Osteoarthritis & $2(5.7)$ & $0(0.0)$ & $9(8.2)$ & $10(10.2)$ \\
\hline Severe renal disease & $1(2.9)$ & $0(0.0)$ & $1(0.9)$ & $4(4.1)$ \\
\hline
\end{tabular}

$H b A 1 c$ glycated hemoglobin, $n$ number of respondents, $P W D$ people with diabetes, $S D$ standard deviation, TIDM type 1 diabetes mellitus, T2DM type 2 diabetes mellitus

awareness, particularly among those with T2DM.

The mean age of Japanese T1DM CGs (Table 2) was $50.3 \pm 10.2$ years and $47.3 \pm 13.7$ years for T2DM. Two T1DM CGs were employed full-time, and one was a homemaker/housewife. Four T2DM CGs were employed full-time, one was employed parttime, and one was unemployed.

\section{Severe Hypoglycemia Characteristics and Actions}

The majority of Japanese PWD had experienced fewer than two SH events in the past 12 months (Table 1), while the mean number of SH events experienced by PWD was approximately $4.3 \pm 6.9(\mathrm{~T} 1 \mathrm{DM})$ and $2.0 \pm 1.3(\mathrm{~T} 2 \mathrm{DM})$ in the past 3 years. PWD and CGs reported that the most recent $\mathrm{SH}$ event occurred at home, with $22.2 \%$ of CGs being present at an SH event in the past 12 months. Overall, $34.3 \%$ (T1DM) and $55.6 \%$ (T2DM) of Japanese PWD had impaired hypoglycemia awareness, based on Gold score $\geq 4$.
In Japanese PWD, most SH events occurred outside the healthcare setting and were unaccompanied by an HCP (Table 3). Overall, most PWD reported their most recent $\mathrm{SH}$ episode occurred at home (68.6\% T1DM, 44.4\% T2DM), with many being alone during the event $(22.9 \%$ T1DM, 44.4\% T2DM). Most SH events occurred in the evening $(40.0 \%)$ or after midnight (31.4\%) for T1DM, and at midday $(33.3 \%)$ or during the afternoon (44.4\%) for T2DM. In addition, Japanese participants with T1DM reported a higher frequency of nocturnal hypoglycemia compared to those with T2DM (51.4\% and $11.1 \%$, respectively) while for the UK PWD nocturnal hypoglycemia was reported by $42.7 \%$ (T1DM) and $27.6 \%(\mathrm{~T} 2 \mathrm{DM})$.

Most Japanese PWD (71.4\% T1DM, 88.9\% T2DM) reported consuming sugars as rescue treatment. For T1DM, in approximately $28.6 \%$ of cases an ambulance was called, which resulted in $90.0 \%$ being transported to a hospital, and approximately half of them arriving in an unconscious state (Table 3$)$. Only 2.9\% $(n=1)$ of Japanese PWD reported receiving glucagon as rescue treatment. The main reported cause of $\mathrm{SH}$ events was eating less than usual or planned 
Table 2 Baseline demographics and clinical characteristics of caregivers in Japan and the UK

\begin{tabular}{|c|c|c|c|c|}
\hline & \multicolumn{2}{|c|}{ Japanese caregivers $(n=9)$} & \multicolumn{2}{|c|}{ UK caregivers $(n=172)$} \\
\hline & $\begin{array}{l}\text { T1DM } \\
(n=3)\end{array}$ & $\begin{array}{l}\text { T2DM } \\
(n=6)\end{array}$ & $\begin{array}{l}\text { T1DM } \\
(n=111)\end{array}$ & $\begin{array}{l}\text { T2DM } \\
(n=61)\end{array}$ \\
\hline Caregiver age, years (SD) & $50.3(10.2)$ & $47.3(13.7)$ & $49.9(12.3)$ & $51.4(13.4)$ \\
\hline Age of PWD being cared for, years (SD) & $61.3(14.0)$ & $60.3(14.4)$ & $44.4(21.5)$ & $65.9(15.0)$ \\
\hline Female caregiver, $n(\%)$ & $2(66.7)$ & $2(33.3)$ & $73(65.8)$ & $39(63.9)$ \\
\hline Time since diagnosis of PWD, years (SD) & $22.3(27.5)$ & $14.2(10.3)$ & $23.2(16.6)$ & $17.7(11.2)$ \\
\hline $\begin{array}{l}\text { PWD had } 1 \mathrm{SH} \text { event in the past } 12 \text { months, } \\
n(\%)\end{array}$ & $3(100)$ & $6(100)$ & $36(32.4)$ & $18(29.5)$ \\
\hline \multicolumn{5}{|l|}{ Caregiver relationship to PWD, $n(\%)$} \\
\hline Spouse/partner & $2(66.7)$ & $3(50.0)$ & $50(45.0)$ & $33(54.1)$ \\
\hline Family member/legal guardian & $1(33.3)$ & $3(50.0)$ & $52(46.8)$ & $27(44.3)$ \\
\hline Parent/legal guardian & $1(33.3)$ & $0(0.0)$ & $31(27.9)$ & $3(4.9)$ \\
\hline Child & $0(0.0)$ & $3(50.0)$ & $16(14.4)$ & $20(32.8)$ \\
\hline \multicolumn{5}{|l|}{ Most recent laboratory HbAlc for PWD, $n(\%)$} \\
\hline$<6.5 \%(48 \mathrm{mmol} / \mathrm{mol})$ & $0(0.0)$ & $0(0.0)$ & $16(14.4)$ & $0(0.0)$ \\
\hline $6.5-7.0 \%(48-53 \mathrm{mmol} / \mathrm{mol})$ & $1(33.3)$ & $2(33.3)$ & $18(16.2)$ & $10(16.4)$ \\
\hline $7.1-8 \%(54-64 \mathrm{mmol} / \mathrm{mol})$ & $0(0.0)$ & $3(50.0)$ & $18(16.2)$ & $15(24.6)$ \\
\hline $8.1-9 \%(65-75 \mathrm{mmol} / \mathrm{mol})$ & $1(33.3)$ & $0(0.0)$ & $9(8.1)$ & $6(9.8)$ \\
\hline$>9.1 \%(>75 \mathrm{mmol} / \mathrm{mol})$ & $1(33.3)$ & $1(16.7)$ & $13(11.7)$ & $7(11.5)$ \\
\hline Unknown & $0(0.0)$ & $0(0.0)$ & $37(33.3)$ & $23(37.7)$ \\
\hline \multicolumn{5}{|l|}{ Location of most recent SH event for PWD, $n$ (\%) } \\
\hline Home & $3(100)$ & $6(100)$ & $97(87.4)$ & $51(83.6)$ \\
\hline Outside of home & $0(0.0)$ & $0(0.0)$ & $14(12.6)$ & $10(16.4)$ \\
\hline \multicolumn{5}{|l|}{ Caregiver employment status, $n(\%)$} \\
\hline Employed, full-time work & $2(66.7)$ & $4(66.7)$ & $52(46.8)$ & $19(31.1)$ \\
\hline Employed, part-time work & $0(0.0)$ & $1(16.7)$ & $14(12.6)$ & $10(16.4)$ \\
\hline Homemaker/housewife & $1(33.3)$ & $0(0.0)$ & $14(12.6)$ & $8(13.1)$ \\
\hline Retired & $0(0)$ & $0(0)$ & $19(17.1)$ & $16(26.2)$ \\
\hline Disabled & $0(0)$ & $0(0)$ & $6(5.4)$ & $4(6.6)$ \\
\hline Unemployed & $0(0.0)$ & $1(16.7)$ & $4(3.6)$ & $2(3.3)$ \\
\hline Other & $0(0)$ & $0(0)$ & $2(1.8)$ & $2(3.3)$ \\
\hline
\end{tabular}


Table 2 continued

\begin{tabular}{llllll}
\hline & \multicolumn{2}{l}{ Japanese caregivers $(\boldsymbol{n}=\mathbf{9})$} & & \multicolumn{2}{l}{ UK caregivers $(\boldsymbol{n}=\mathbf{1 7 2})$} \\
\cline { 2 - 3 } & $\begin{array}{l}\text { T1DM } \\
(\boldsymbol{n}=\mathbf{3})\end{array}$ & $\begin{array}{l}\text { T2DM } \\
(\boldsymbol{n}=\mathbf{6})\end{array}$ & & $\begin{array}{l}\text { T1DM } \\
(\boldsymbol{n}=\mathbf{1 1 1})\end{array}$ & $\begin{array}{l}\text { T2DM } \\
(\boldsymbol{n}=\mathbf{6 1})\end{array}$ \\
\hline $\begin{array}{l}\text { Typical work schedule for PWD (if employed), } \\
n(\%)\end{array}$ & $n=1$ & $n=1$ & $n=66$ & $n=29$ \\
Morning shift & $0(0.0)$ & $0(0.0)$ & $5(7.6)$ & $2(6.9)$ \\
Afternoon shift & $0(0.0)$ & $1(100)$ & $1(1.5)$ & $1(3.4)$ \\
Evening/night shift & $1(100)$ & $0(0.0)$ & $3(4.5)$ & $1(3.4)$ \\
Regular rotation between shifts & $0(0.0)$ & $0(0.0)$ & $8(12.1)$ & $3(10.3)$ \\
Regular daytime hours (09:00 to 17:00) & $0(0.0)$ & $0(0.0)$ & & $46(69.7)$ & $20(69.0)$ \\
Other & $0(0.0)$ & $0(0.0)$ & $3(4.5)$ & $2(6.9)$ \\
\hline
\end{tabular}

$H b A 1 c$ glycated hemoglobin, $n$ number of respondents, $P W D$ people with diabetes, $S D$ standard deviation, $S H$ severe hypoglycemia, $T 1 D M$ type 1 diabetes mellitus, T2DM type 2 diabetes mellitus

(48.6\% T1DM, 11.1\% T2DM); 20.0\% (T1DM) and $44.4 \%$ (T2DM) did not remember or were unaware of the cause of the event.

\section{Conversations Around and Impact of Most Recent Severe Hypoglycemia Event}

Overall, 25.0\% (T1DM) and 28.6\% (T2DM) of participants did not discuss their most recent SH with an HCP before their next scheduled appointment. Of participants who had a discussion with an HCP regarding a recent $\mathrm{SH}$ event, the majority waited until their next appointment with a doctor $(75.0 \%$ T1DM, $71.4 \%$ T2DM). Overall, $71.4 \%$ (T1DM) and $100.0 \%$ (T2DM) of participants reported that their doctor's appointment occurred within a month of the SH event, $14.3 \%$ (T1DM) did not see a doctor until at least a month after the event, with another $14.3 \%$ (T1DM) unable to recall the timing of their doctor's appointment (Table 3). For the UK cohort, 43.2\% (T1DM) and $32.7 \%$ (T2DM) of PWD saw their HCPs and discussed their SH events within a week of the event.

The major behavioral modifications made among Japanese PWD following the most recent $\mathrm{SH}$ event included carrying sugar/carbohydrates $(68.6 \%$ T1DM, $55.6 \%$ T2DM $)$, checking blood glucose levels more often (37.1\% T1DM, 33.3\% T2DM), and modifications of insulin therapy (31.4\% T1DM, 22.2\% T2DM). There were no reports of a glucagon kit being obtained after the event. For the majority of Japanese PWD, the reported reason glucagon was not used during the most recent SH event was that the prescription was not available or filled (68.8\% T1DM, 44.4\% T2DM) (Table 4).

\section{Behavioral and Emotional Impact of Most Recent Severe Hypoglycemia Event}

SH had a behavioral and emotional impact on both PWD and CGs (Fig. 2). After the most recent $\mathrm{SH}$ event, Japanese participants reported feeling scared $(85.7 \% \mathrm{~T} 1 \mathrm{DM}, 55.6 \% \mathrm{~T} 2 \mathrm{DM})$, unprepared $(62.9 \% \mathrm{~T} 1 \mathrm{DM}, 77.8 \% \mathrm{~T} 2 \mathrm{DM})$, and helpless (60.0\% T1DM, 33.3\% T2DM). According to Japanese PWD, SH events affected various aspects of their lives including physical activity (40.0\% T1DM, 55.6\% T2DM), mood or emotional state $(42.9 \% \mathrm{~T} 1 \mathrm{DM}, 33.3 \% \mathrm{~T} 2 \mathrm{DM})$, and daily activities (40.0\% T1DM, 22.2\% T2DM).

The majority of CGs reported an emotional impact after witnessing an SH event with many feeling scared $(100.0 \%$ for both T1DM and T2DM), unprepared (100.0\% T1DM, 50.0\% 
Table 3 Characteristics of most recent severe hypoglycemia event

\begin{tabular}{|c|c|c|c|c|}
\hline \multirow[t]{2}{*}{ Events/actions, $n(\%)$} & \multicolumn{2}{|c|}{ Japanese PWD $(n=44)$} & \multicolumn{2}{|c|}{ UK PWD $(n=208)$} \\
\hline & T1DM & T2DM & T1DM & T2DM \\
\hline Companion during last $\mathrm{SH}$ event & $n=35$ & $n=9$ & $n=110$ & $n=98$ \\
\hline Alone & $8(22.9)$ & $4(44.4)$ & $24(21.8)$ & $30(30.6)$ \\
\hline Spouse/partner & $14(40.0)$ & $2(22.2)$ & $68(61.8)$ & $52(53.1)$ \\
\hline Parent/legal guardian & $7(20.0)$ & $1(11.1)$ & $3(2.7)$ & $0(0.0)$ \\
\hline Child & $1(2.9)$ & $0(0.0)$ & $2(1.8)$ & $1(1.0)$ \\
\hline Other & $5(14.3)$ & $2(22.2)$ & $13(11.8)$ & $15(15.3)$ \\
\hline Location of most recent $\mathrm{SH}$ event & $n=35$ & $n=9$ & $n=110$ & $n=98$ \\
\hline Home & $24(68.6)$ & $4(44.4)$ & $97(88.2)$ & $75(76.5)$ \\
\hline Work & $2(5.7)$ & $2(22.2)$ & $5(4.5)$ & $4(4.1)$ \\
\hline Walking on the street & $3(8.6)$ & $1(11.1)$ & $1(0.9)$ & $3(3.1)$ \\
\hline In a car/bus/train & $2(5.7)$ & $1(11.1)$ & $0(0.0)$ & $2(2.0)$ \\
\hline On a trip (business or vacation) & $1(2.9)$ & $0(0.0)$ & $3(2.7)$ & $1(1.0)$ \\
\hline At a mall/shopping center & $1(2.9)$ & $0(0.0)$ & $1(0.9)$ & $6(6.1)$ \\
\hline Other/do not remember & $2(5.8)$ & $1(11.1)$ & $3(2.7)$ & $7(7.1)$ \\
\hline Timing of $\mathrm{SH}$ event & $n=35$ & $n=9$ & $n=110$ & $n=98$ \\
\hline Morning & $3(8.6)$ & $1(11.1)$ & $11(10.0)$ & $13(13.3)$ \\
\hline Midday & $2(5.7)$ & $3(33.3)$ & $4(3.6)$ & $12(12.2)$ \\
\hline Afternoon & $4(11.4)$ & $4(44.4)$ & $23(20.9)$ & $29(29.6)$ \\
\hline Evening & $14(40.0)$ & $1(11.1)$ & $25(22.7)$ & $16(16.3)$ \\
\hline After midnight & $11(31.4)$ & $0(0.0)$ & $44(40.0)$ & $28(28.6)$ \\
\hline Do not know & $1(2.9)$ & $0(0.0)$ & $3(2.7)$ & $0(0.0)$ \\
\hline Asleep during $\mathrm{SH}$ event & $n=35$ & $n=9$ & $n=110$ & $n=98$ \\
\hline Yes & $18(51.4)$ & $1(11.1)$ & $47(42.7)$ & $27(27.6)$ \\
\hline No & $16(45.7)$ & $7(77.8)$ & $60(54.5)$ & $67(68.4)$ \\
\hline Do not know/remember & $1(2.9)$ & $1(11.1)$ & $3(2.7)$ & $4(4.1)$ \\
\hline Actions taken during $\mathrm{SH}$ event & $n=35$ & $n=9$ & $n=110$ & $n=98$ \\
\hline Ate/drank sugars & $25(71.4)$ & $8(88.9)$ & $95(86.4)$ & $91(92.9)$ \\
\hline Received glucagon & $1(2.9)$ & $0(0.0)$ & $11(10.0)$ & $5(5.1)$ \\
\hline Other & $1(2.9)$ & $1(11.1)$ & $6(5.5)$ & $7(7.1)$ \\
\hline Do not know/remember & $2(5.7)$ & $0(0.0)$ & $0(0.0)$ & $1(1.0)$ \\
\hline Went to hospital without calling ambulance & $2(5.7)$ & $1(11.1)$ & $1(0.9)$ & $1(1.0)$ \\
\hline Called ambulance & $10(28.6)$ & $0(0.0)$ & $14(12.7)$ & $9(9.2)$ \\
\hline
\end{tabular}


Table 3 continued

\begin{tabular}{|c|c|c|c|c|}
\hline \multirow[t]{2}{*}{ Events/actions, $n$ (\%) } & \multicolumn{2}{|c|}{ Japanese PWD $(n=44)$} & \multicolumn{2}{|c|}{ UK PWD $(n=208)$} \\
\hline & T1DM & T2DM & T1DM & T2DM \\
\hline & $n=10$ & $n=0$ & $n=14$ & $n=9$ \\
\hline \multirow[t]{2}{*}{ Transported to hospital } & $9(90.0)$ & - & $7(50.0)$ & $5(55.6)$ \\
\hline & $n=9$ & $n=0$ & $n=7$ & $n=5$ \\
\hline Arrived at hospital unconscious & $5(55.6)$ & - & $0(0.0)$ & $0(0.0)$ \\
\hline Arrived at hospital disoriented & $1(11.0)$ & - & $4(57.1)$ & $4(80.0)$ \\
\hline Admitted overnight & $3(33.3)$ & - & $2(28.6)$ & $3(60.0)$ \\
\hline Cause of most recent $\mathrm{SH}$ event & $n=35$ & $n=9$ & $n=110$ & $n=98$ \\
\hline Accidentally injected the wrong insulin & $2(5.7)$ & $0(0.0)$ & $4(3.6)$ & $0(0.0)$ \\
\hline Accidentally took too much insulin/incorrect dose & $4(11.4)$ & $1(11.1)$ & $21(19.1)$ & $11(11.2)$ \\
\hline Took too much insulin to correct hyperglycemia & $4(11.4)$ & $0(0.0)$ & $9(8.2)$ & $2(2.0)$ \\
\hline Drank alcohol & $1(2.9)$ & $1(11.1)$ & $4(3.6)$ & $7(7.1)$ \\
\hline Ate less than planned/usual & $17(48.6)$ & $1(11.1)$ & $27(24.5)$ & $33(33.7)$ \\
\hline A work or social event prevented eating as planned & $1(2.9)$ & $3(33.3)$ & $1(0.9)$ & $3(3.1)$ \\
\hline Injected insulin, but food not served when expected & $0(0.0)$ & $1(11.1)$ & $6(5.5)$ & $7(7.1)$ \\
\hline Exercised more than planned or realized & $4(11.4)$ & $1(11.1)$ & $18(16.4)$ & $20(20.4)$ \\
\hline Had an illness & $4(11.4)$ & $0(0.0)$ & $3(2.7)$ & $7(7.1)$ \\
\hline Other & $1(2.9)$ & $0(0.0)$ & $9(8.2)$ & $6(6.1)$ \\
\hline Do not know/remember & $7(20.0)$ & $4(44.4)$ & $26(23.6)$ & $21(21.4)$ \\
\hline Conversations regarding $\mathrm{SH}$ event with $\mathrm{HCP}$ & $n=35$ & $n=9$ & $n=110$ & $n=98$ \\
\hline \multicolumn{5}{|l|}{ Discussed with HCP } \\
\hline Yes & $31(88.6)$ & $7(77.8)$ & $87(79.1)$ & $80(81.6)$ \\
\hline No & $3(8.6)$ & $1(11.1)$ & $7(6.4)$ & $9(9.2)$ \\
\hline Do not know/remember & $1(2.9)$ & $1(11.1)$ & $16(14.5)$ & $9(9.1)$ \\
\hline \multicolumn{5}{|l|}{ Discussed most recent $\mathrm{SH}$ event with $\mathrm{HCP}$} \\
\hline Yes, in office visit & $27(77.1)$ & $7(77.8)$ & $31(28.2)$ & $41(41.8)$ \\
\hline Yes, via telephone & $0(0.0)$ & $0(0.0)$ & $11(10.0)$ & $7(7.1)$ \\
\hline Yes, via email & $1(2.9)$ & $0(0.0)$ & $0(0.0)$ & $1(1.0)$ \\
\hline No & $6(17.1)$ & $2(22.2)$ & $65(59.1)$ & $45(45.9)$ \\
\hline Do not know/other & $1(2.9)$ & $0(0.0)$ & $3(2.7)$ & $4(4.1)$ \\
\hline Timing of conversation regarding most recent $\mathrm{SH}$ event & $n=28$ & $n=7$ & $n=44$ & $n=52$ \\
\hline Within a week of the event & $4(14.3)$ & $1(14.3)$ & $19(43.2)$ & $17(32.7)$ \\
\hline
\end{tabular}


Table 3 continued

\begin{tabular}{|c|c|c|c|c|}
\hline \multirow[t]{2}{*}{ Events/actions, $n$ (\%) } & \multicolumn{2}{|c|}{ Japanese PWD $(n=44)$} & \multicolumn{2}{|c|}{ UK PWD $(n=208)$} \\
\hline & T1DM & T2DM & T1DM & T2DM \\
\hline More than a week after the event & $1(3.6)$ & $1(14.3)$ & $11(25.0)$ & $12(23.1)$ \\
\hline Do not know/remember & $2(7.1)$ & $0(0.0)$ & $2(4.5)$ & $2(3.8)$ \\
\hline At the next doctor appointment & $21(75.0)$ & $5(71.4)$ & $12(27.3)$ & $21(40.4)$ \\
\hline $\begin{array}{l}\text { Timing of doctor appointment after most } \\
\text { recent severe low blood sugar event }\end{array}$ & $n=21$ & $n=5$ & $n=12$ & $n=21$ \\
\hline Within a month & $15(71.4)$ & $5(100.0)$ & $3(25.0)$ & $10(47.6)$ \\
\hline More than a month & $3(14.3)$ & $0(0.0)$ & $6(50.0)$ & $10(47.6)$ \\
\hline Do not know/remember & $3(14.3)$ & $0(0.0)$ & $3(25.0)$ & $1(4.8)$ \\
\hline Actions after most recent $\mathrm{SH}$ event & $n=35$ & $n=9$ & $n=110$ & $n=98$ \\
\hline Changed insulin or timing or dose of insulin & $11(31.4)$ & $2(22.2)$ & $37(33.6)$ & $25(25.5)$ \\
\hline Carried sugar/something sweet/changed meal plan & $24(68.6)$ & $5(55.6)$ & $59(53.6)$ & $56(57.1)$ \\
\hline Wore a CGM device & $3(8.6)$ & $0(0.0)$ & $5(4.5)$ & $0(0.0)$ \\
\hline Checked blood glucose more often & $13(37.1)$ & $3(33.3)$ & $57(51.8)$ & $50(51.0)$ \\
\hline Researched more information & $3(8.6)$ & $0(0.0)$ & $0(0.0)$ & $9(9.2)$ \\
\hline Obtain glucagon kits or kept a kit nearby & $0(0.0)$ & $0(0.0)$ & $5(4.5)$ & $1(1.0)$ \\
\hline Other & $2(5.7)$ & $1(11.1)$ & $9(8.2)$ & $7(7.1)$ \\
\hline
\end{tabular}

$C G M$ continuous glucose monitoring, $H C P$ healthcare provider, $n$ number of respondents, $P W D$ people with diabetes, $S H$ severe hypoglycemia, TIDM type 1 diabetes mellitus, T2DM type 2 diabetes mellitus

T2DM), and helpless (66.7\% T1DM, 83.3\% T2DM).

\section{DISCUSSION}

$\mathrm{SH}$ is a serious complication for PWD. It is therefore vital to understand $\mathrm{SH}$ experiences and related behaviors from the perspectives of Japanese PWD and CGs. Most published studies evaluate $\mathrm{SH}$ as a common adverse event in people with T1DM or T2DM treated with insulin and/or insulin secretagogues. In the current study, we specifically recruited individuals who experienced at least one $\mathrm{SH}$ event in the prior 3 years while being treated with only insulin. This study design resulted in challenges regarding participant recruitment, and hence a relatively small sample size was obtained. Conversely, using data from UK PWD as a reference population, we report the first real-world data concerning $\mathrm{SH}$ events, combined with reported behaviors, and experiences among Japanese PWD and CGs.

The annual incidence of $\mathrm{SH}$ is low, with a survey of 346,939 patients conducted by the Japan Diabetes Society revealing a total of 2237 patients who experienced $\mathrm{SH}$, an incidence rate of $0.64 \%$ [3]. As shown in Table 1, 25 out of 44 participants with T1DM (71\%), and 4 out of 9 Japanese participants with insulin-treated T2DM (44\%), reported at least one SH event in the past 12 months. Regarding adults with insulin-treated T2DM, Ando et al. [15] reported 
Table 4 Reasons why glucagon was not used during the most recent severe hypoglycemia event among patients who did not use glucagon

\begin{tabular}{|c|c|c|c|c|}
\hline \multirow[t]{2}{*}{$\operatorname{Reason}^{\mathrm{a}}(\%)$} & \multicolumn{2}{|c|}{ Japanese PWD $(n=41)$} & \multicolumn{2}{|c|}{ UK PWD $(n=191)$} \\
\hline & $\begin{array}{l}\text { T1DM } \\
(n=32)\end{array}$ & $\begin{array}{l}\text { T2DM } \\
(n=9)\end{array}$ & $\begin{array}{l}\text { T1DM } \\
(n=99)\end{array}$ & $\begin{array}{l}\text { T2DM } \\
(n=92)\end{array}$ \\
\hline Glucagon was not readily available & $3(9.4)$ & $0(0.0)$ & $21(21.2)$ & $20(21.7)$ \\
\hline No prescription available or filled & $22(68.8)$ & $4(44.4)$ & $34(34.3)$ & $45(48.9)$ \\
\hline Not aware of glucagon & $0(0)$ & $0(0)$ & $3(3.0)$ & $15(16.3)$ \\
\hline Glucagon kit had expired & $0(0)$ & $0(0)$ & $2(2.0)$ & $0(0)$ \\
\hline Glucagon was available but called ambulance & $0(0)$ & $0(0)$ & $1(1.0)$ & $0(0)$ \\
\hline Glucagon was available, but other treatment worked & $1(3.1)$ & $0(0.0)$ & $19(19.2)$ & $3(3.3)$ \\
\hline $\begin{array}{l}\text { Was alone when the severe low blood sugar event } \\
\text { occurred }\end{array}$ & $1(3.1)$ & $3(33.3)$ & $8(8.1)$ & $5(5.4)$ \\
\hline Other & $3(9.4)$ & $1(11.1)$ & $12(12.1)$ & $4(4.3)$ \\
\hline Do not know/remember & $3(9.4)$ & $1(11.1)$ & $10(10.1)$ & $12(13.0)$ \\
\hline
\end{tabular}

$n$ number of respondents, PWD people with diabetes, TIDM type 1 diabetes mellitus, T2DM type 2 diabetes mellitus

${ }^{\mathrm{a}}$ Multiple responses were possible

no SH events over 24 weeks in a population of 40 adults with well-controlled T2DM who were taking insulin plus either canagliflozin or liraglutide. Similarly, Kadowaki et al. [16] reported no SH events at 52 weeks among 184 adults with insulin-treated T2DM and with poor glycemic control. Abiru et al. [17] reported SH events among 528 participants aged at least 26 years from the Study of Adults' Glycemia in T1DM; in this T1DM population, only $5.5 \%$ reported experiencing one or more $\mathrm{SH}$ events within the previous 6 months. As reported in the literature [3, 15-17], Japan has low rates of $\mathrm{SH}$ in people with T2DM and T1DM. Furthermore, in the current study, there were limited numbers of people who met the inclusion criteria for enrollment. This report focuses exclusively on the experiences of those who satisfied the eligibility criteria.

A key finding of our analysis is that $\mathrm{SH}$ most often occurred outside the healthcare setting in Japan, mostly at home, and was accompanied by a spouse/partner or other CG. Following the most recent SH event, many Japanese PWD did not make immediate contact with their doctor or other HCP. For those Japanese PWD who discussed their $\mathrm{SH}$ event with their doctor, this mostly occurred at the next doctor's appointment up to a month after the event. Discussion of $\mathrm{SH}$ events with HCPs within a week of the event was numerically higher among participants from the UK versus Japan. Further research is warranted regarding this finding, which may include a recommendation for HCP input in the immediate response to $\mathrm{SH}$ events in the Japan PWD.

An important finding is the reported emotional toll of SH events in the Japan cohort, with many PWD and CGs feeling scared, unprepared, and helpless. The emotional impact appears to be high in Japanese PWD and CGs. Given this emotional impact, and the fact that Japan PWD seemed less inclined to consult an HCP shortly after the event, the development of educational programs and awareness of SH in Japan may improve the clinical environment for patients at elevated risk of SH. Our findings suggest a need to better educate and support Japanese PWD and CGs regarding SH 


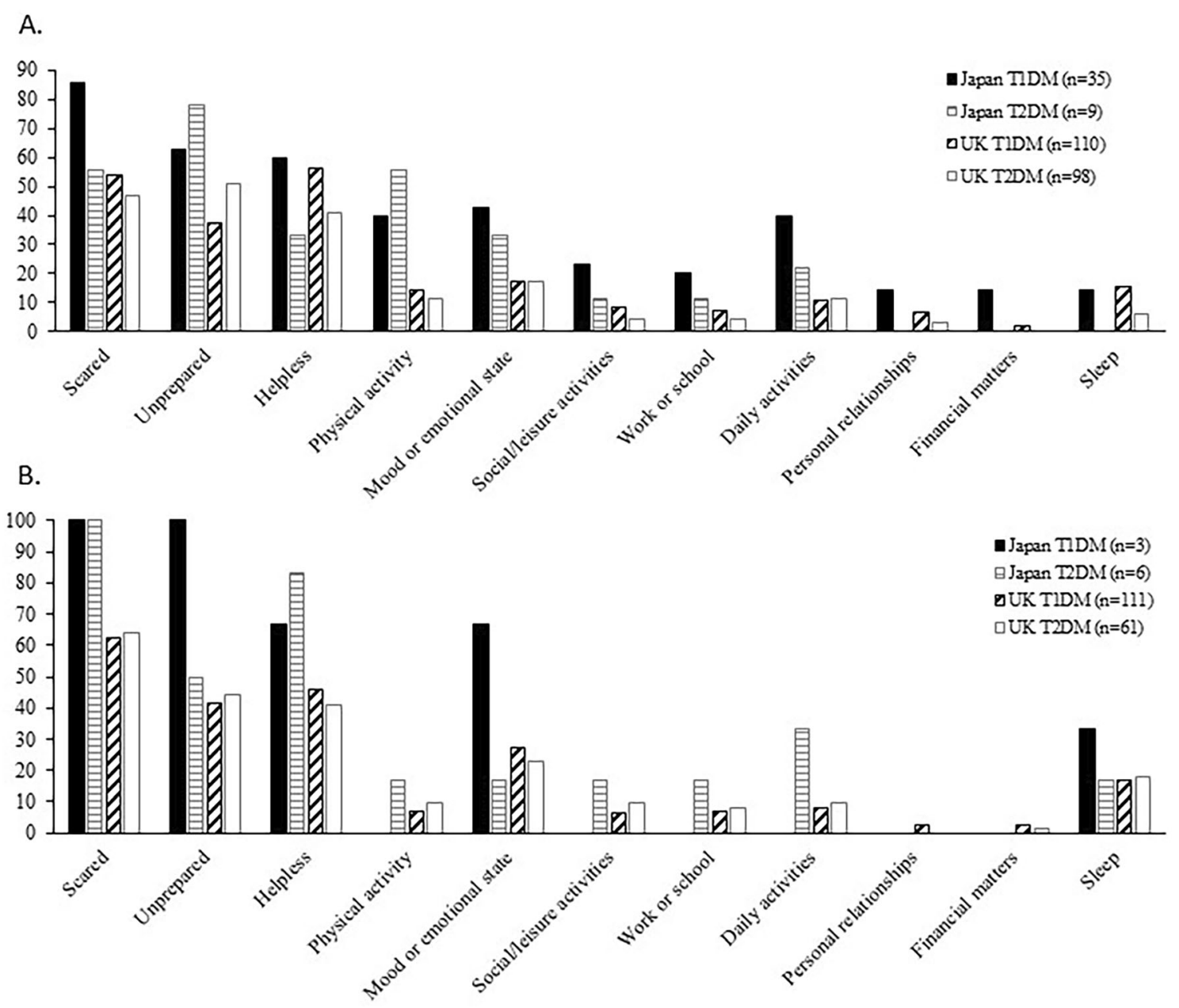

Fig. 2 Effect of severe hypoglycemia events on PWD on multiple life domains. Data presented are the percentage of PWD (a) and caregivers (b) in Japan and the UK that felt the most recent severe hypoglycemic event affected

prevention and management, but further studies are warranted.

Conversations about SH between Japanese HCPs and PWD appear to be insufficient. Some participants (8.6\% T1DM, 11.1\% T2DM) reported that they have never discussed $\mathrm{SH}$ with an HCP and a greater proportion reported they did not discuss their most recent $\mathrm{SH}$ event with an HCP (17.1\% T1DM, 22.2\% T2DM). Patients who underreport $\mathrm{SH}$ to their HCP not only impede opportunities to improve diabetes management but they are also at a higher risk of future $\mathrm{SH}$ events [18]. In alignment with the causes of $\mathrm{SH}$ outlined by the Japanese Diabetes Society [3], emotional status and multiple life domains. $n$ number of participants, PWD people with diabetes, T1DM type 1 diabetes mellitus, T2DM type 2 diabetes mellitus, $U K$ United Kingdom

common reasons for $\mathrm{SH}$ events in Japanese study participants included diet, illness, exercise, and misuse of insulin. Many common causes of SH may be avoided by frequent and SH-specific discussions between Japanese PWD and HCPs. Additional benefits to improved communication may include improved diabetes management and thereby a reduced risk of $\mathrm{SH}$, which is likely to extend to improvements in quality of life and an easing of economic burden.

Similar to the UK and Canadian [14] cohorts, Japanese PWD often consume sugar in order to recover from an $\mathrm{SH}$ event when possible. 
However, rescue treatment of $\mathrm{SH}$ in Japan has some notable differences in comparison to other countries. While emergency ambulance services incur substantial costs to patients in many countries, this service incurs no fees to users in Japan (as well as the UK). The ambulance service in Japan arrives on average within $8.7 \mathrm{~min}$ of an emergency call, with a mean handover time of $39.5 \mathrm{~min}$ to hospital staff [19] (compared with a median handover time of 75 min for emergency SH events in the UK [20]). In the current study, a considerable proportion of Japanese participants with T1DM called an ambulance for an SH event. This may be due to the convenience of the ambulance service in Japan (free of charge and quick arrival/handover time). As a result of the limited number of Japanese participants in this study, further research is warranted.

Glucagon appears to be currently underutilized by Japanese PWD at high risk for $\mathrm{SH}$, and glucagon possession rates are indeed low compared with other countries [21]. Nasal glucagon was launched in Japan in October 2020, and is expected to provide an important treatment option for the emergency treatment of $\mathrm{SH}$ in Japanese PWD [22]. However, in comparison to other countries, ambulance staff in Japan have limited options for treating SH. Legally, ambulance personnel in Japan are permitted to administer intravenous glucose injections under physician supervision but are not able to administer glucagon. This may contribute to the high proportion of Japanese PWD (T1DM) who reported being transported to hospital for an SH event in the current study. In the UK, most SH cases can be successfully treated at the scene by emergency ambulance staff [20], hence the numerically lower proportion of patients transported to hospital and subsequently admitted for $\mathrm{SH}$ treatment.

Many Japanese PWD reported that their most recent SH occurred at home. Previous findings suggest raising awareness of the importance of earlier intervention outside the hospital setting is essential [12]. Furthermore, the international DAWN2 study demonstrated that Japanese PWD showed high participation rates in diabetes education programs; however, they also indicated substantial concerns regarding hypoglycemia [23]. Hence, there appears room for improvement in education regarding the prevention of $\mathrm{SH}$, and the available treatment options for SH events in Japan, especially for high-risk PWD and their CGs. Such educational programs may improve preparation for future $\mathrm{SH}$ episodes and help alleviate fears and concerns regarding $\mathrm{SH}$ events. SH still occurs despite advancements in the development of new generation blood glucose-lowering medications, insulin-delivery devices, and continuous glucose monitors. Hence, the management of $\mathrm{SH}$ needs to continue to progress.

A limitation of the CRASH study is that it relied on respondents' memories of events occurring up to 3 years prior, creating potential recall bias. Additionally, the study design utilized an online survey, which may have excluded those with limited computer skills or access. The number of Japanese participants was limited, and the findings in this population may not represent the entire diabetes population. Although the number of participants was limited, similarly low numbers of SH among Japanese PWD are reported in other studies [3, 15-17]. As the majority of study participants had T1DM, caution should be employed when generalizing these findings to people with T2DM. The limited number of Japanese participants may also be due to the lower prevalence of $\mathrm{SH}$ in Asian populations compared with Western countries. However, this is the first study to describe patient-reported $\mathrm{SH}$ and experiences from PWD and CGs in an Asian population. Furthermore, $\mathrm{SH}$ was evaluated as the main focus rather than as an adverse event of diabetes treatment. Despite these limitations, our findings are consistent with those previously reported, and provide real-world data with practical relevance to $\mathrm{SH}$ experiences in the Japanese population.

\section{CONCLUSIONS}

Our analysis of the CRASH study data has highlighted numerous important findings regarding the experiences of Japanese PWD and CGs surrounding $\mathrm{SH}$ events. Our findings 
suggest that many $\mathrm{SH}$ events occur at home, and many Japanese PWD and CGs are highly affected emotionally by SH events. Despite this emotional burden, effective communication between PWD and HCPs appears to be lacking, with most Japanese PWD waiting until the next doctor's appointment to discuss their most recent $\mathrm{SH}$ event. Our findings also indicate that an ambulance service is commonly used in an $\mathrm{SH}$ event, but that few PWD use glucagon as rescue therapy. Education regarding $\mathrm{SH}$ treatment options may improve treatment preparation for SH events and may help to alleviate patient concerns. Our results provide important insight into conversations, actions, and preparedness surrounding $\mathrm{SH}$ that can be applied to PWD and CGs and inform clinical decisionmaking and public health policy.

\section{ACKNOWLEDGEMENTS}

Funding. This study was sponsored by Eli Lilly and Company. The journal's Rapid Service Fee was funded by Eli Lilly Japan K.K.

Authorship. All named authors meet the International Committee of Medical Journal Editors (ICMJE) criteria for authorship for this article, take responsibility for the integrity of the work as a whole and have given their approval for this manuscript to be published.

Author Contributions. Beth Mitchell and Frank Snoek created the study concept and design; Erik Spaepen and Mark Peyrot analyzed data; and data interpretation was performed by Toshiyuki Iwahori, Yukiko Nagai, Mark Peyrot, and Beth Mitchell.

Medical Writing, Editorial, and Other Assistance. Medical writing and editorial assistance were provided by Lisa Cossens and Dana Schamberger of Syneos Health and funded by Eli Lilly Japan K.K. We thank those who participated in this study and Shingo Kojima for his dedication to participant recruitment.
Disclosures. Frank Snoek reports advisory roles with Abbott, Eli Lilly and Company, Novo Nordisk, and Roche Diabetes; consultant roles with Eli Lilly and Company and Novo Nordisk; research support from Sanofi and Novo Nordisk; and speaker honoraria from Eli Lilly and Company, Novo Nordisk, and Servier. Toshiyuki Iwahori and Yukiko Nagai are employees of Eli Lilly Japan K.K. Beth Mitchell is an employee of Eli Lilly and Company. Yukiko Nagai and Beth Mitchell are shareholders of Eli Lilly and Company. Erik Spaepen is a paid consultant working for Eli Lilly and Company. Mark Peyrot reports advisory roles with Calibra, Eli Lilly and Company, and Novo Nordisk; and speaker honoraria from Eli Lilly and Company.

Compliance with Ethics Guidelines. The study protocol was approved by Eli Lilly and Company. Informed consent was obtained from all individual participants included in the study. This study was performed in accordance with the Helsinki Declaration of 1964 and its later amendments.

Data Availability. Eli Lilly and Company provides access to all individual participant data collected during the trial, after anonymization, with the exception of pharmacokinetic or genetic data. Data are available to request 6 months after the indication studied has been approved in the USA and EU and after primary publication acceptance, whichever is later. No expiration date of data requests is currently set once data are made available. Access is provided after a proposal has been approved by an independent review committee identified for this purpose and after receipt of a signed data sharing agreement. Data and documents, including the study protocol, statistical analysis plan, clinical study report, and blank or annotated case report forms, will be provided in a secure data sharing environment. For details on submitting a request, see the instructions provided at www.vivli.org.

Open Access. This article is licensed under a Creative Commons Attribution-NonCommercial 4.0 International License, which permits any non-commercial use, sharing, adaptation, 
distribution and reproduction in any medium or format, as long as you give appropriate credit to the original author(s) and the source, provide a link to the Creative Commons licence, and indicate if changes were made. The images or other third party material in this article are included in the article's Creative Commons licence, unless indicated otherwise in a credit line to the material. If material is not included in the article's Creative Commons licence and your intended use is not permitted by statutory regulation or exceeds the permitted use, you will need to obtain permission directly from the copyright holder. To view a copy of this licence, visit http://creativecommons.org/licenses/by$\mathrm{nc} / 4.0 /$.

\section{REFERENCES}

1. Kovatchev B, Cobelli C. Glucose variability: timing, risk analysis, and relationship to hypoglycemia in diabetes. Diabetes Care. 2010;39:502-10.

2. Iqbal A, Heller S. Managing hypoglycaemia. Best Pract Res Clin Endocrinol Metab. 2016;30:413-30.

3. Namba M, Iwakura T, Nishimura R, et al. Diabetes Society Committee for Surveys on Severe Hypoglycemia. The current status of treatment-related severe hypoglycemia in Japanese patients with diabetes mellitus: a report from the committee on a survey of severe hypoglycemia in the Japan Diabetes Society. J Diabetes Investig. 2018;9:642-56.

4. Seaquist ER, Anderson J, Childs B, et al. Hypoglycemia and diabetes: a report of a workgroup of the American Diabetes Association and The Endocrine Society. Diabetes Care. 2013;36:1384-95.

5. Johnston SS, Conner C, Aagren M, Smith DM, Bouchard J, Brett J. Evidence linking hypoglycemic events to an increased risk of acute cardiovascular events in patients with type 2 diabetes. Diabetes Care. 2011;34:1164-70.

6. Desouza CV, Bolli GB, Fonseca V. Hypoglycemia, diabetes, and cardiovascular events. Diabetes Care. 2010;33:1389-94.

7. International hypoglycaemia study group. Hypoglycaemia, cardiovascular disease, and mortality in diabetes: epidemiology, pathogenesis, and management. Lancet Diabetes Endocrinol. 2019;7: 385-96.
8. Whitmer RA, Karter AJ, Yaffe K, Quesenberry CP Jr, Selby JV. Hypoglycemic episodes and risk of dementia in older patients with type 2 diabetes mellitus. JAMA. 2009;301:1565-72.

9. McCoy RG, Van Houten HK, Ziegenfuss JY, Shah ND, Wermers RA, Smith SA. Increased mortality of patients with diabetes reporting severe hypoclycemia. Diabetes Care. 2012;35:1897-901.

10. Bloomgarden Z. Fear of hypoglycemia. J Diabetes. 2016;9:108-10.

11. Goto A, Noda M, Inoue M, Goto M, Hadrien C. Increasing number of people with diabetes in Japan: is this trend real? Intern Med. 2016;55: 1827-30.

12. Tanaka M, Ito $H$, Kusano E, et al. Medical expenses of diabetes patients delivered by emergency transportation due to severe hypoglycemia. J Jpn Diab Soc. 2019;62:9-16 (in Japanese).

13. Rossi MC, Nicolucci A, Ozzello A, et al. Impact of severe and symptomatic hypoglycemia on quality of life and fear of hypoglycemia in type 1 and type 2 diabetes. Results of the Hypos-1 observational study. Nutr Metab Cardiovasc Dis. 2019;29: 736-43.

14. Mojdami D, Mitchell BD, Spaepen E, et al. Conversations and reactions around severe hypoglycemia study: results in Canadian adults with insulin-treated diabetes and their caregivers. Can J Diabetes. 2021;45:236-42.

15. Ando Y, Shigiyama F, Hirose T, Kumashiro N. Simplification of complex insulin regimens using canagliflozin or liraglutide in patients with wellcontrolled type 2 diabetes: a 24 -week randomized controlled trial. J Diabetes Investig. 2021;12: 1816-26.

16. Kadowaki T, Seino Y, Kaku K, et al. A randomized, placebo-controlled study to evaluate the efficacy and safety of adding omarigliptin to insulin therapy in Japanese patients with type 2 diabetes and inadequate glycaemic control. Diabetes Obes Metab. 2021;23:1242-51.

17. Abiru N, Shimada A, Nishimura R, et al. Glycemic control status, diabetes management patterns, and clinical characteristics of adults with type 1 diabetes in Japan: Study of Adults' Glycemia in T1DM subanalysis. Diabetol Int. 2021;12:460-73.

18. Ratzki-Leewing A, Black JE, Mequanint S, et al. The 'unspoken' truth: suboptimal reporting of severe hypoglycemia in diabetes (InHypo-DM study). Can J Diabetes. 2018;42:S52-3. 
19. Japan Fire and disaster management agency. Rescue treatment statistics (Fiscal year: Reiwa 1): https:// www.fdma.go.jp/publication/rescue/items/kkkg_ r01_01_kyukyu.pdf. Accessed on 28 August 2020 (in Japanese).

20. Khunti K, Fisher H, Paul S, Iqbal M, Davies MJ, Siriwardena AN. Severe hypoglycaemia requiring emergency medical assistance by ambulance services in the East Midlands: a retrospective study. Prim Care Diabetes. 2013;7:159-65.

21. Murata T, Okazaki K, Yanagisawa K, et al. Glucagon underutilized among type 1 diabetes mellitus patients in Japan. Diabetes Technol Ther. 2013;15: 748-50.

22. Matsuhisa M, Takita Y, Nasu R, Nagai Y, Ohwaki K, Nagashima $H$. Nasal glucagon as a viable alternative for treating insulin-induced hypoglycaemia in Japanese patients with type 1 or type 2 diabetes: a phase 3 randomized crossover study. Diab Obes Metab. 2020;22:1167-75.

23. Nicolucci A, Kovacs Burns K, Holt RIG, et al. Diabetes attitudes, wishes and needs second study (DAWN2): cross-national benchmarking of diabetes-related psychosocial outcomes for people with diabetes. Diabet Med. 2013;30:767-77. 